\title{
EL-SHELLING ON COMODERNISTIC LATTICES
}

\author{
TIANSI LI
}

\begin{abstract}
We prove the equivalence of EL-shellability and the existence of recursive atom ordering independent of roots. We show that a comodernistic lattice, as defined by Schweig and Woodroofe, admits a recursive atom ordering independent of roots, therefore is EL-shellable. We also present and discuss a simpler EL-shelling on one of the most important classes of comodernistic lattice, the order congruence lattices.
\end{abstract}

\section{INTRODUCTION}

Modernistic and Comodernistic lattices are two large classes of finite lattices with shellable order complexes. Schweig and Woodroofe defined and studied these lattices in 22 and showed that a wide range of lattices are either modernistic or comodernistic, including subgroup lattices of finite solvable groups, supersolvable and left-modular lattices, semi-modular lattices, $k$-equal partition lattices, order congruence lattices, and others. They proved in 2] that comodernistic lattices are CL-shellable, which implies that the order complexes of modernistic and comodernistic lattices are shellable.

In this paper, we show that comodernistic lattices are EL-shellable, as defined in 1. This can be viewed as a generalization of Woodroofe's result on subgroup lattices. After Shareshian showed in [3] that subgroup lattices of solvable groups are CL-shellable, Woodroofe proved a stronger result in 5 that these lattices are in fact EL-shellable.

It is shown in [1] that a poset is CL-shellable if and only if it admits a recursive atom ordering. We will prove a similar result for EL-shellable posets in order to prove our main result.

Recall that a recursive atom ordering of $P$ assigns to each pair $(x, r)$ with $x \in P \backslash\{\hat{1}\}$ and a maximal chain $r$ in $[\hat{0}, x]$ an ordering of the atoms in $[x, \hat{1}]$

Lemma 1.1. If $P$ admits a recursive atom ordering such that, for each pair $(x, r)$, the atom ordering of $[x, \hat{1}]$ does not depend on $r$, then $P$ is EL-shellable.

The following is our main result.

Theorem 1.2. Comodernistic lattices are EL-shellable.

We will further study the class of order congruence lattices after proving Theorem 1.2. We will show that these lattices are EL-shellable using integer labels without invoking Theorem 1.2.

In the next section, we will include necessary definitions concerning modernistic and comodernistic lattices. For readers unfamiliar with EL-shellability and CLshellability, we recommend [1] for more information.

Department of Mathematics and Statistics, Washington University in St. Louis. 


\section{Preliminaries}

We call a simplicial complex $\Delta$ shellable if there is an ordering of its facets $F_{1}, F_{2}, \ldots, F_{t}$ such that $\left(\bigcup_{i=1}^{k} F_{i}\right) \cap F_{k+1}$ is pure and $\left(\operatorname{dim}\left(F_{k}\right)-1\right)$-dimensional for $k=1,2, \ldots, t-1$. A poset $P$ is shellable if its order complex $\Delta P$ is.

For a poset $P$, an edge-labeling is a map from the edge set of the Hasse diagram of $P$ to some label poset $\Lambda$. An edge-labeling is an $E L-$ shelling if for every interval $[x, y]$ of $P$, there exists a unique weakly increasing maximal chain lexicographically preceding all other maximal chains in $[x, y]$ in a fixed linear extension of the poset $\Lambda$. A chain-edge labeling is a map from the set of all pairs $(c, e)$ to the label set $\Lambda$, where $c$ is a maximal chain of $P$ and $e$ is an edge in $c$, such that $(c, e)$ and $\left(c^{\prime}, e\right)$ get the same label if $c$ and $c^{\prime}$ coincide from $\hat{0}$ to $e$. A poset $P$ is $C L-$ shellable if it admits a chain-edge labeling such that for every interval $[x, y]$ and maximal chain $r$ in $[\hat{0}, x]$, in the rooted interval $[x, y]_{r}$, there exists a unique weakly increasing maximal chain lexicographically preceding all other maximal chains in a fixed linear extension of the label poset $\Lambda$.

Clearly, EL-shellability implies CL-shellability. More importantly, both ELshellability and CL-shellability imply shellabiliy.

Theorem 2.1. [1, Proposition 2.3] EL-shellability $\Rightarrow C L$-shellability $\Rightarrow$ Shellability.

Another notion commonly used in the context of lexicographic shellability is recursive atom ordering.

Definition 2.2. 4, Definition 4.2.1] A poset $P$ is said to admit a recursive atom ordering if the length of $P$ is 1 or if the length of $P$ is greater than 1 and there is an ordering $a_{1}, a_{2}, \ldots, a_{t}$ of the atoms of $\mathrm{P}$ which satisfies:

(1) For all $j=1,2, \ldots, t,\left[a_{j}, \hat{1}\right]$ admits a recursive atom ordering in which the atoms of $\left[a_{j}, \hat{1}\right]$ that come first in the ordering are those that cover some $a_{i}$, where $i<j$.

(2) For all $i<j$, if $a_{i}, a_{j}<y$ then there is a $k<j$ and an atom $z$ of $a_{j}$ such that $z<y$ and $a_{k}<z$.

Bjöner and Wachs have proved that CL-shellability is equivalent to the existence of a recursive atom ordering.

Theorem 2.3. 4, Theorem 4.2.2] A bounded poset $P$ admits a recursive atom ordering if and only if $P$ is $C L$-shellable.

Next we introduce modernistic and comodernistic lattices. Let $L$ denote a lattice. Recall that an element $m$ in $L$ is left-modular if for any $x<y$ in $L$, we have $(x \vee m) \wedge y=x \vee(m \wedge y)$. A lattice $L$ is modernistic if for every interval of $L$, there exists a left-modular atom in that interval. A lattice is comodernistic if it is the dual of a modernistic lattice. That is, there exists a left-modular coatom in every interval. Schweig and Woodroofe proved that comodernistic lattices are CL-shellable [2].

Theorem 2.4. [2, Theorem 1.2] If $L$ is a comodernistic lattice, then $L$ has a $C L$ labeling

As stated above in Theorem 1.2, we will show that comodernistic lattices are EL-shellable. To show that comodernistic lattices are EL-shellable, we need the notion of a sub-M-chain, which can be viewed as an analogy of an M-chain in a left-modular lattice. 
Definition 2.5. 2] A maximal chain $\hat{0}=m_{0} \lessdot m_{1} \lessdot \cdots \lessdot m_{n}=\hat{1}$ in $L$ is a sub-M-chain if for every $i$, the element $m_{i}$ is left-modular in the interval [ $\left[\hat{0}, m_{i+1}\right]$.

We also list here two lemmas from 2] that will help prove our main theorem. We refer readers to [2] for the proofs of the lemmas.

Lemma 2.6. 2, Lemma 3.1] Let $L$ be a lattice with a sub-M-chain $\boldsymbol{m}$ of length $n$. Then no chain of $L$ has length greater than $n$.

Lemma 2.7. [2, Lemma 2.12] Let $m$ be a coatom of the lattice $L$. Then $m$ is left-modular in $L$ if and only if for every $y$ such that $y \not \leq m$ we have $m \wedge y \lessdot y$.

\section{Proof of Lemma 1.1}

Let $P$ be a CL-shellable poset with an induced recursive atom ordering such that for each $x \in P \backslash\{\hat{1}\}$, all orderings of the atoms of $[x, \hat{1}]$ are the same, as in Lemma 1.1. We will construct an EL-labeling for $P$. For every edge $e=[x, y]$ in the Hasse diagram of $P$, we define a $\left(\kappa_{e}+1\right)$-tuple, where $\kappa_{e}$ is the number of maximal chains in $[\hat{0}, x]$. The last coordinate records the edge itself. That is, for $e=[x, y]$, let the last coordinate of the label of $e$ simply be the 2-tuple (x,y). Now we consider the first $\kappa_{e}$ coordinates. These are indexed by the roots in $[x, y]$. Call these the $\kappa_{e}$ large coordinates of the label. In each large coordinate, we place a 2-tuple, whose first entry is the union of the corresponding root and the edge itself, and the second entry is the label induced by the root in the CL-shelling of $P$. Let us call these 2 -tuples the small coordinates. We order the $\kappa_{e}$ large coordinates according to the original CL-labeling of $P$. That is, if $C$ is lexicographically the $k^{\text {th }}$ maximal chain in $[\hat{0}, x]$ according to the CL-labeling, the $k^{\text {th }}$ large coordinate of $e$ consists of $C \cup e$ and the label induced by $C$.

Now we define a partial order on the labeling set. Suppose $e=[x, y]$ and $e^{\prime}=$ $\left[x^{\prime}, y^{\prime}\right]$ are two edges in the Hasse diagram labeled as above. Then we say $e \leq e^{\prime}$ if $y<x^{\prime}$, or if $y=x^{\prime}$ and for some large coordinate $e_{m}$ of $e$, there exists a large coordinate $e_{n}^{\prime}$ of $e^{\prime}$ such that $e_{m} \leq e_{n}^{\prime}$, by which we mean that the root (first small coordinate) of $e_{m}$ is contained in the root of $e_{n}^{\prime}$, and the label (second small coordinate) in $e_{m}$ is less than or equal to the label in $e_{n}^{\prime}$.

Let us first check that this is a well-defined partial order. Obviously we have reflexivity. Antisymmetry is satisfied because if $e<e^{\prime}, x$ cannot be above or equal $y^{\prime}$. Transitivity holds because if $e<e^{\prime}$ and $e^{\prime}<e^{\prime \prime}, y<x^{\prime \prime}$. So this is indeed a partial order.

We now check that this edge-labeling gives an EL-shelling of $P$. For any interval $[x, y]$, let $C$ be the weakly-increasing chain in the original CL-labeling (with respect to any roots). We claim that the new edge-labeling on $P$ makes $C$ the unique weakly increasing and lexicographic first maximal chain in the interval.

The fact that $C$ is weakly-increasing follows from the consistency of the CLlabeling. Let $C$ be weakly increasing in $[x, y]_{r}$ for all roots $r$. Suppose there exists some $C^{\prime}=\left\{c_{0} \lessdot c_{1} \lessdot \cdots \lessdot c_{k}\right\}$ in $[x, y]$ that is also weakly increasing in the new edge-labeling. Then for each $0<i<k$, there exists a root $r_{i}$ such that $c_{i}$ is the first atom in $\left[c_{i-1}, c_{i+1}\right]_{r_{i}}$. Notice that the assumption on the atom orderings implies that whether labels of two consecutive edges in $\left[c_{i-1}, c_{i+1}\right]$ are weakly increasing is independent of roots. Hence $C^{\prime}$ must be weakly increasing in some rooted interval, which would further imply that there are two weakly increasing maximal chains in 
one rooted interval. This contradicts to $P$ being CL-shellable. A similar argument shows that $C$ must be the lexicographic first maximal chain of the interval.

\section{Proof of Theorem 1.2}

Let $L$ be a comodernistic lattice. We fix a sub-M-chain $\mathbf{m}=\left\{\hat{0}=m_{0} \lessdot m_{1} \lessdot\right.$ $\left.\ldots \lessdot m_{n}=\hat{1}\right\}$ of $L$ and prove Theorem 1.2 by induction on height. For each atom $a$ of $L$, label $[\hat{0}, a]$ by the index of the minimal element of $\mathbf{m}$ that is larger than or equal to $a$ in the partial order. Suppose we have labeled all elements of height less than $k$. For $y$ of height $k$, we pick an element $z$ covered by $y$ and define a sub-M-chain $\mathbf{m}_{y}$ in $[y, \hat{1}]$ as follows. Let $w$ be the minimal element above $y$ in $\mathbf{m}_{z}$, where $\mathbf{m}_{z}$ has been chosen by induction, and pick some sub-M-chain of $[y, w]$. Then $\mathbf{m}_{y}$ is the sub-M-chain of $[y, w]$ followed by the rest of the sub-M-chain of $\mathbf{m}_{z}$. We label the atom edges of $[y, \hat{1}]$ using $\mathbf{m}_{y}$ the same way we label atom edges of $L$ with m.

Now we show that the edge labeling defined above induces a recursive atom ordering independent of roots as in Lemma 1.1. We claim that any atom ordering that is consistent with the edge labeling is a recursive atom ordering. That is, if for a fixed linear extension, the label of $[x, y]$ precedes the label of $\left[x, y^{\prime}\right]$, where $y$ and $y^{\prime}$ both cover $x$, then $y$ precedes $y^{\prime}$ in the atom ordering of $x$. And if the label of $[x, y]$ equals the label of $\left[x, y^{\prime}\right]$, we can either have $y$ precedes $y^{\prime}$ or $y^{\prime}$ precedes $y$. We prove this claim by induction on coheight. It is obvious for all intervals $[x, \hat{1}]$ where $x$ is a coatom of $L$. Suppose the claim stands for all $[x, \hat{1}]$ of length less than $k$. For any $[x, \hat{1}]$ of length $k$, consider any atom $a_{i}$ of the interval.

$\left[a_{i}, \hat{1}\right]$ admits a recursive atom ordering by induction. For any two atoms $b_{j}$ and $b_{k}$ of $\left[a_{i}, \hat{1}\right]$, suppose $j<k$ and $b_{k}$ covers some $a_{l}$ with $l<i$. We need to show that $b_{j}$ covers some $a_{m}$ for $m<i$. Since $b_{k}$ covers $a_{i}$ and $a_{l}$ with $l<i, b_{k} \in[x, w]$ where $w$ is the minimal element above $a_{i}$ in the sub-M-chain $\mathbf{m}_{x}$ of $[x, \hat{1}]$. Therefore $b_{j}<w$ since $j<k$. Let $w^{\prime}$ be the maximal element in $\mathbf{m}_{x}$ below $w$ and not above $b_{j}$. Then $b_{j} \wedge w^{\prime}$ is an atom in $[x, \hat{1}]$ prior to $a_{i}$ and covered by $b_{j}$.

Now for any two atoms $a_{i}$ and $a_{j}$ with $i<j$ of $[x, \hat{1}]$, we need to find an atom of $\left[a_{j}, \hat{1}\right]$ that covers some $a_{k}<a_{i} \vee a_{j}$ with $k<j$. Consider the first atom $b$ in $\left[a_{j}, \hat{1}\right]$ that sits below $a_{i} \vee a_{j}$. Let $w$ be the minimal element of $\mathbf{m}_{x}$ that sits above $a_{i} \vee a_{j}$. Then $w$ is the minimal element of $\mathbf{m}_{x}$ that sits above $a_{j}$ since $i<j$. Let $w^{\prime}$ be the element in $\mathbf{m}_{x}$ covered by $w$. Then $b \wedge w^{\prime}$ is an atom in $[x, \hat{1}]$ that is prior to $a_{j}$

\section{Order Congruence Lattices}

The order congruence lattice $\mathcal{O}(P)$ of a poset $P$ is the set of all equivalence classes of level set partitions from $P$ to $\mathbb{Z}$. That is, the set of all weakly order preserving maps from $P$ to $\mathbb{Z}$, where two such maps are considered equivalent if they induce the same partition on $P$.

For example, the order congruence lattice on a totally ordered set is a boolean lattice. The order congruence lattice on a set of pairwise incomparable elements is isomorphic to a partition lattice. In general, order congruence lattice of any poset can be considered as in between the boolean lattice and the partition lattice. 


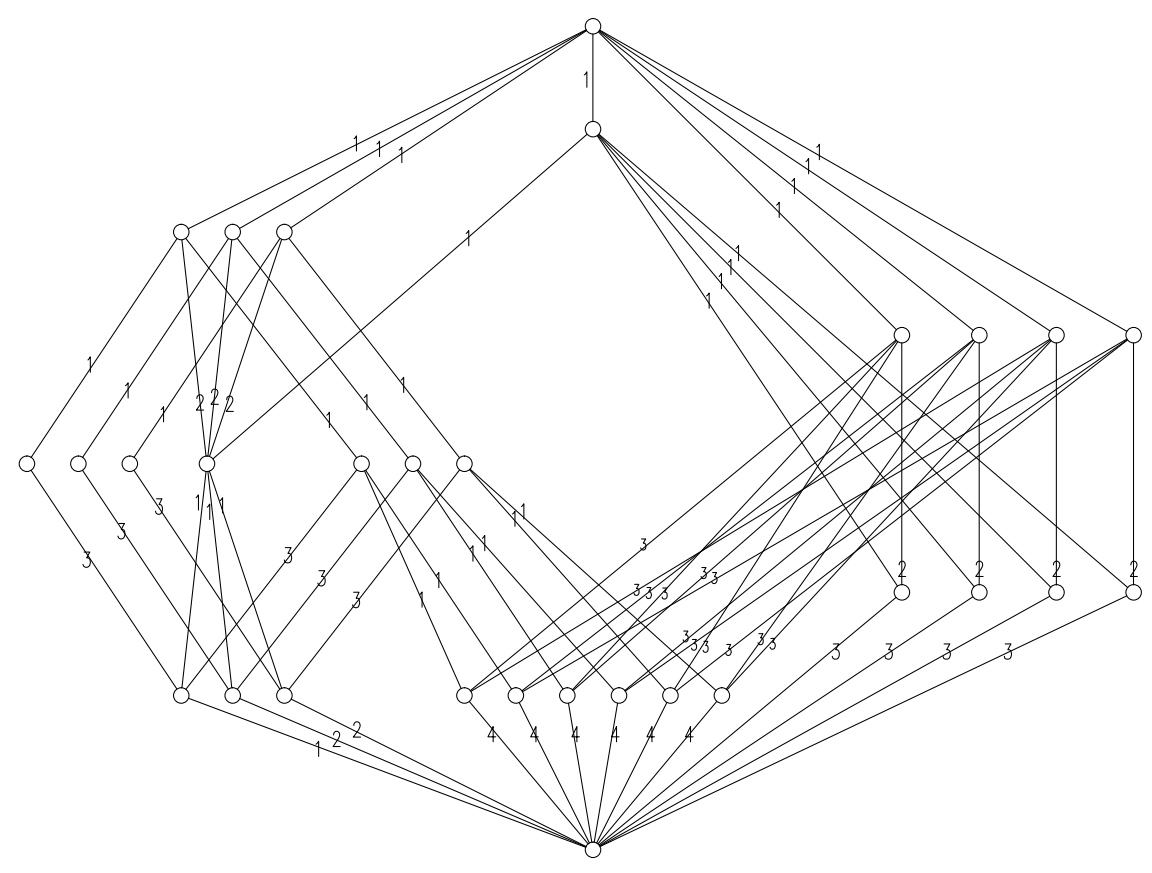

Figure 1 . Initial labeling on $S_{4}$ given by sub-M-chain

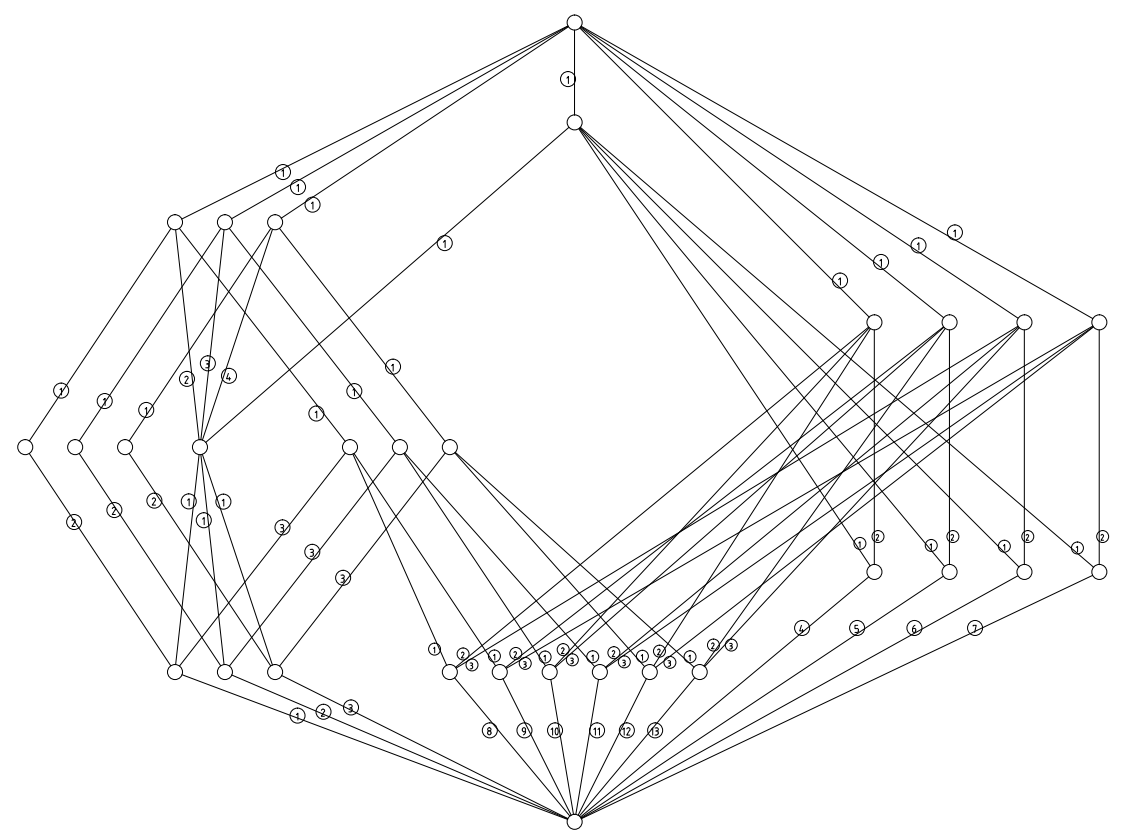

FiguRe 2. Root-independent recursive atom ordering from the initial labeling 


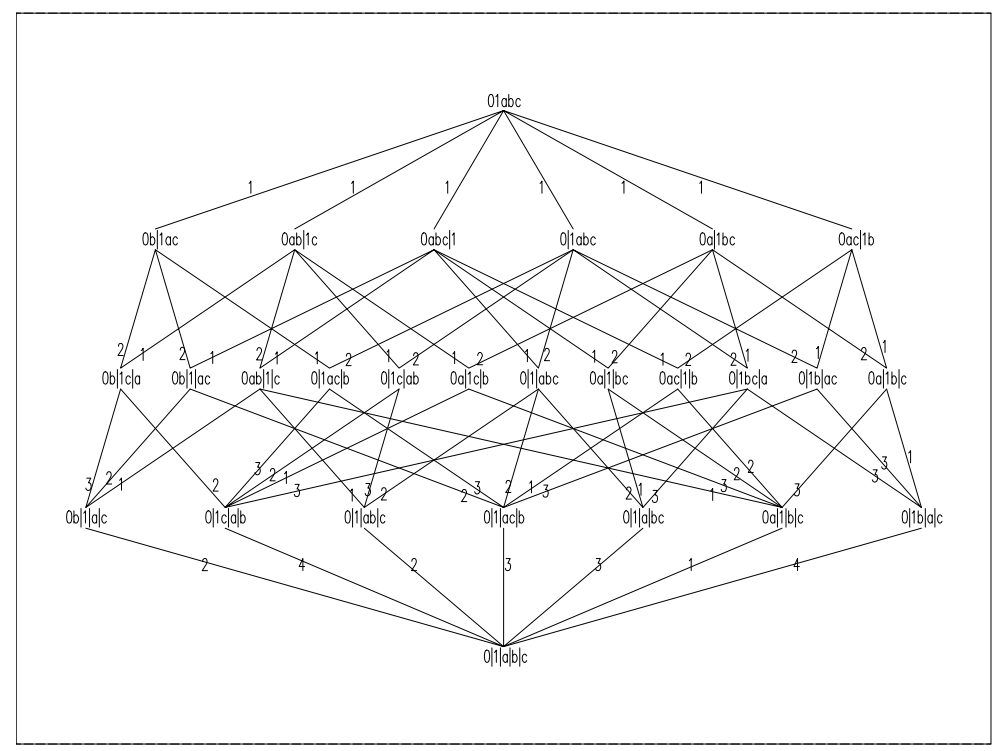

FiguRE 3. EL-shelling on $N_{5}$ with linear extension $\hat{0} \rightarrow a \rightarrow b \rightarrow$ $c \rightarrow \hat{1}$

Schweig and Woodroofe proved in [2] that order congruence lattices are comodernistic, therefore CL-shellable. We here present a different proof where any linear extension of $P$ gives a sub-M-chain and an EL-shelling on $\mathcal{O}(P)$.

Fix a linear extension of $P=\left\{z_{1}, z_{2}, \ldots, z_{n}\right\}$. For an element in $\mathcal{O}(P)$ with $k$ blocks, we can index each block as follows. First we take the smallest element in the linear extension of each block as representative. We assign indices 1 through $k$ to the blocks preserving the order of their representatives. And we use these indices to construct a sub-M-chain and EL-shelling as follows.

Let $C$ be the maximal chain $\hat{0}=c_{1} \lessdot c_{1} \lessdot \cdots \lessdot c_{n}=\hat{1}$ where $c_{k}$ is obtained by having the first $k$ elements $\left(z_{1}\right.$ through $\left.z_{k}\right)$ in one block, and then one block each for the remaining elements.

Proposition 5.1. $C$ is a sub-M-chain which induces an EL-shelling on $\mathcal{O}(P)$.

Proof. For any $k$ and $x \in\left[\hat{0}, c_{k}\right]$, we need to show either $x<c_{k-1}$ or $x \wedge c_{k-1} \lessdot x$. If $z_{k}$ in $x$ is a block by itself, $x<c_{k-1}$. Otherwise, $x \wedge c_{k-1}$ is obtained by isolating $z_{k}$ to a single block from $x$. Hence $x \wedge c_{k-1}$ is covered by $x$ and $C$ is a sub-M-chain.

Now we show that it induces an EL-shelling on $\mathcal{O}(P)$. Consider the following edge-labeling. For any edge $[x, y]$, if $y$ is obtained from $x$ by merging the $i^{t h}$ block and the $j^{t h}$ block where $i<j$, we assign $j$ to $[x, y]$. This is a well-defined edgelabeling. Now we show that it is an EL-shelling.

Consider any interval $[x, y]$ in $\mathcal{O}(P)$. Notice that if $y$ consists of $k$ blocks, then any edge in the interval can be viewed as a merge within one of the $k$ blocks of $y$. The lexicographically first maximal chain is obtained by consecutively merging the 
smallest indexed two sub-blocks in the same block of $y$. This is a weakly increasing chain. Next we check that this is the unique weakly increasing chain of the interval. We show that any other chain must either violate the merging order within a block of $y$ or among blocks of $y$. Suppose we merge two sub-blocks within a block of $y$ that are not the two smallest possible blocks to merge. Merging with a smaller sub-block later will result in a smaller edge label, in which case the maximal chain cannot be weakly increasing. Suppose we missed a merge within a smaller block of $y$. The edge obtained by completing that missed merge will again create a smaller label hence the maximal chain cannot be weakly increasing.

\section{REFERENCES}

[1] Anders Björner and Michelle Wachs. On lexicographically shellable posets. Transactions of the American Mathematical Society, 277(1):323-341, 1983.

[2] Jay Schweig and Russ Woodroofe. A broad class of shellable lattices. Advances in Mathematics, 313:537-563, 2017.

[3] John Shareshian. On the shellability of the order complex of the subgroup lattice of a finite group. Transactions of the American Mathematical Society, 353(7):2689-2703, 2001.

[4] Michelle L Wachs. Poset topology: tools and applications. arXiv preprint math/0602226, 2006.

[5] Russ Woodroofe. An el-labeling of the subgroup lattice. Proceedings of the American Mathematical Society, 136(11):3795-3801, 2008. 